\title{
8 Verbindlichkeit im Verbund erzielen oder: Von den Schwierigkeiten, Ärzte zu einen
}

\author{
Hans-Jürgen Beckmann und Claudia Schrewe
}

Ärzte gelten nicht zu Unrecht als Individualisten und sind im Rahmen ihrer Praxistätigkeit bisher gewohnt, alleine für ihre Belange zu kämpfen. Dort, wo in der Vergangenheit Verträge mit Kostenträgern zur Bezahlung ihrer Leistungen geschlossen werden mussten, wurden und werden die meisten von ihnen nach wie vor von den kassenärztlichen Vereinigungen vertreten. Aber die Zeiten ändern sich rasant und Krankenkassen können seit jüngstem direkt Verträge mit Leistungserbringern bzw. deren Verbünden unter Umgehung der kassenärztlichen Vereinigungen schließen. Der Trend weg von den bisherigen Kollektivverträgen hin zu Selektivverträgen ist mittlerweile eindeutig zu erkennen.

Die Ärzte selbst reagieren auf diese veränderten Rahmenbedingungen unter anderem mit Gründung neuer Zusammenschlüsse, den Ärztenetzen, die sich den Kostenträgern als Vertragspartner anbieten. Weit über 350 Ärztenetze gibt es bundesweit mittlerweile. Ihr Organisationsgrad reicht von Vereinen bis zu Aktiengesellschaften mit einem Trend zu Kapitalgesellschaften und Genossenschaften.

Ob Kapitalgesellschaft oder Cenossenschaft, ob Verein oder Aktiengesellschaft, allen gemein ist, dass Vertragsabschlüsse dieser ärztlichen Zusammenschlüsse hohe Anforderungen an die Verlässlichkeit ihrer Mitglieder stellen. Verbindlichkeit ist von ihnen gefordert, denn nur gemeinsam können vereinbarte Vertragsinhalte eingehalten werden. Die Einung der Netzmitglieder in der Umsetzung geschlossener Verträge stellt daher große Anforderungen an das Netzmanagement! 
Was muss also das Management eines ärztlichen Verbundes tun, um seine Mitglieder für seine Visionen und Ziele zu gewinnen, und wie hält man die Mitglieder dauerhaft im Verbund? Basierend auf den konkreten Erfahrungen in der alltäglichen Arbeit des 73-köpfigen Ärztenetzes „Medizin und Mehr“, kurz „MuM“, in Ostwestfalen versuchen die Autoren, diese Frage zu beantworten. Ein Patentrezept gibt es nicht, gilt es doch Besonderheiten jeder Region, ob Stadt ob Land, ob hohe Krankenhausdichte, usw. bei der Motivation der Mitglieder zu berücksichtigen. Ergänzend werden Diskussionsergebnisse aus der Beratung für und mit anderen Netzen eingebunden. Wir danken den Kollegen für die konstruktive Zusammenarbeit im Rahmen unserer Netzmanagertreffen. Beteiligt waren Verbünde aus Südbrandenburg, Wehye, Thüringen, Lippe, Kassel, Solingen, Leverkusen, Ludwigshafen, Lennetal, Amberg, Marburg und Bünde.

\section{Das ideale Ärztenetz}

\section{Das ideale Ärztenetz lässt sich wohl so beschreiben: Die Mitglieder nehmen dauerhaft am Netzgeschehen teil, sie sind über relevante Er- eignisse informiert, sie identifizieren sich mit den Zielen des Netzes, und sie sind motiviert, ihren Beitrag zur Netzarbeit zu leisten.}

Ein Arzt identifiziert sich aber zunächst mit seiner Profession und nicht mit einer Organisationsform oder einer Gruppe, eine Organisation lässt sich am treffendsten mit „Bewerkstelligung“ übersetzen und bedeutet: Planung und Durchführung eines Vorhabens.

Eine Gruppe hingegen muss so etwas wie eine Gruppenidentität, ein WirGefühl entwickelt haben. Das soziale Zusammenleben innerhalb der Gruppe ist geprägt durch dauerhafte soziale Beziehungen und Kontakte, durch Eigenund Zusammenhandeln, durch Einheit sozial Handelnder mit gemeinsamen Werten und Interessen, durch Unmittelbarkeit von Beziehungen, durch wechselseitige Wahrnehmung der Beteiligten, durch Anwesenheit und direkte Interaktion sowie durch aufeinander abgestimmte soziale Rollen.

Das Wir-Gefühl ist ein wesentlicher, konstituierender Ausgangsfaktor für den Erhalt und Bestand von Gruppen, denn dieses Gruppengefühl gründet in den Gefühlen von Zugehörigkeit und Zusammengehörigkeit.

Der „normale“ Netzarzt muss seine Werte, mit denen er sich identifizieren kann, im Katalog der „Netzwerte“ wieder finden können. Nur so kann Identifikation stattfinden, die ihn motiviert, sich dem Verbund anzuschließen, wobei nicht genug betont werden kann, dass Haltung und Erwartungen des Einzelnen sehr unterschiedlich sind. Interessierte finden hierzu weitere Arbeiten bei der Wirtschafts- und Sozialwissenschaftlichen Fakultät der Friedrich-Alexander-Universität Erlangen.

Das Gesundheitswesen gehört zu den Branchen, in denen sich derzeit viel verändert: Unzählige Reformen erzeugen Planungsunsicherheit und Ängste, 
insbesondere bei denen, die wie die Ärzte in dem sich ändernden System weiter ihr Einkommen erzielen müssen. Es gibt innovative Abenteuer unter Ärzten, aber unter geschildertem Szenario ist es nicht weiter verwunderlich, dass Ärzte mit Veränderungswillen oder gar Visionen eher selten zu finden sind. Häufiger spielen tradiertes Sicherheitsdenken und der Wunsch nach Orientierung eine Rolle, wenn es darum geht, sich einem Netz anzuschließen. Manch einer gibt zudem als Motivation, einem Verbund beizutreten an, lediglich soziale Kontakte pflegen zu wollen. Auch hier geht es darum, Sicherheit und Geborgenheit zu erreichen.

Diese nur kurz skizzierten Motive spielen insbesondere in der Gestaltung der Ansprache der Ärzte eine wesentliche Rolle. Fehler in der Kommunikation können weitreichende Folgen haben: Die Verletzung eines Sicherheitsbedürfnisses durch zu viele und zu schnelle Innovationen kann ebenso zur innerlichen Abkehr des Gruppenmitglieds führen, wie Stagnation verbunden mit dem Gefühl, dass nötige Schritte von einem zögerlichen Management nicht gegangen werden. Diese unterschiedliche Motivationslage und die Tatsache, dass ein Arzt sich mit seiner Profession und nicht mit der Organisationsform identifiziert, macht es den leitenden Akteuren nicht leicht. Oft aber wird die Klage über die mangelnde Kooperationsbereitschaft von Ärzten vorgeschoben, um von Managementfehlern abzulenken: Die Forderung muss lauten, dass der Nutzen der Organisation für den Einzelnen deutlich wird, um die notwendige Partizipation und Motivation zu erzeugen.

Zusammenfassend lässt sich folgendes Ziel formulieren, um die nötige Verbindlichkeit in einem Verbund zu erreichen:

\section{Es müssen Werte geschaffen werden, über die sich jedes Mitglied mit dem Verbund identifizieren kann.}

Im Folgenden werden Maßnahmen dargestellt, um dieses Ziel zu erreichen. Sie sind als Worklist für all diejenigen anzusehen, die sich funktionierende Netzwerke zur Aufgabe gemacht haben.

\section{Gruppenidentifikationsfaktor Nummer 1: Erfolge zeigen!}

Wer gehört nicht gern zu einem erfolgreichen Unternehmen? So gilt es, mit Projekten zu starten, die möglichst schnell Erfolg dokumentieren. Exemplarisch kann hier die gemeinsame Organisation eines regionalen Notfalldienstes genannt werden, da mit relativ wenig Aufwand hoher Nutzen für die Mitglieder darstellbar ist. Eben über einen solchen Erfolg können dann bezüglich größerer Vorhaben Skeptiker ins Boot geholt werden. 


\section{Gruppenidentifikationsfaktor Nummer 2: Nutzen generieren!}

Die Organisation nutzt dem Einzelnen, wenn das Management in der Lage ist, in unsicheren Zeiten Orientierung und Sicherheit zu geben. Der Verbund vertritt berufsständische Interessen gegenüber der Kassenärztlichen Vereinigung, dem oder den Krankenhäusern und zunehmend gegenüber Krankenkassen. Sicherheit meint auch materielle Aspekte. So kommt der Erschließung von neuen Einnahmequellen, z. B. in Form von Selektivverträgen mit Krankenkassen, eine wesentliche Bedeutung zu. Aber auch hierbei gilt, Perspektiven aufzuzeigen, die verlässlich sind. Dementsprechend sind eventuelle Risiken abzusichern. Im Falle der Selektivverträge bedeutet dies konkret, die Beteiligung an der kollektivvertraglichen Versorgung zu erhalten bzw. die Rückkehr hierzu offen zu halten.

\section{Gruppenidentifikationsfaktor Nummer 3: Kontinuität wahren!}

Es gibt also keine schlechten Netzärzte, sondern nur ein schlechtes Management? Die Autoren favorisieren eine andere Betrachtungsweise: Es gibt nur Fehler in der Herangehensweise. Dies begründet sich zunächst erst einmal darin, dass bis heute viele Netzvorstände neben ihrer Praxistätigkeit für ihre Verbünde arbeiten. Die Zeit für konstruktive Arbeiten ist knapp, die Zielfindung schwierig und die Vermittlung dessen, was bei den Arbeitssitzungen entschieden worden ist, bleibt meist als erstes auf der Strecke. Hieraus muss sich die Forderung ableiten, das Management von Arztverbünden insofern zu professionalisieren, als dass diese Aufgabe nicht mehr nebenberuflich und dann auch noch unentgeltlich stattfindet. Eine bezahlte Freistellung ist hier denkbar. Favorisiert werden sollte jedoch eine Lösung, bei der betriebswirtschaftliche Aufgaben von einer nichtärztlichen, angestellten Geschäftsführung in Kooperation mit einer bezahlten, ärztlichen Netzleitung bearbeitet werden. So ergänzen sich betriebswirtschaftliches und ärztliches Know-how, eine wichtige Voraussetzung für Verhandlungen mit Krankenkassen. Die hauptamtliche Geschäftsführung stellt die nötige Kontinuität bei der Erfüllung diffiziler Managementaufgaben sicher.

\section{Gruppenidentifikationsfaktor Nummer 4: Transparenz erzeugen!}

Teilnehmen kann nur der, der auch weiß, worum es geht. Und Transparenz entsteht nur da, wo regelmäßig informiert wird. Hier sind unterschiedliche Wege denkbar, wie z. B. die regelmäßige Information per Newsletter, Rundschreiben oder E-Mail. Es muss jedoch auch eine Partizipation der Beteiligten in Form von Kleingruppenarbeit oder Einsatz von Multiplikatoren, z. B. med. Fachangestellte, umgesetzt werden. Hier steckt der Teufel im Detail und jeder Netzmanager kann an dieser Stelle nur dazu aufgefordert sein, eine möglichst dezidierte Aufstellung möglicher Tools zu erstellen. 
- regelmäßige Information über Arbeitsergebnisse und Neuigkeiten

- Schaffung nachvollziehbarer Strukturen

- Definition eindeutiger Zuständigkeiten

\section{Gruppenidentifikationsfaktor Nummer 5: Kommunikation gestalten!}

Die Kommunikation als Königsweg für Transparenz hat eine Schlüsselfunktion für erfolgreiche Ärztevereinigungen. Die Regeln:

- Information muss über jeden relevanten Arbeitschritt/Entwicklung stattfinden.

- Hierbei müssen die Motive der Netzmitglieder Berücksichtigung finden.

- Es sollten nur Erfolge und Dinge, die sicher sind, kommuniziert werden.

- Alle Sachverhalte sind möglichst positiv darzustellen.

- Wiederholungen und Honorierung jeder Beteiligung von anderen sind wichtig.

- Es bedarf der klaren Positionierung zu aktuellen berufspolitischen Ereignissen, rasch, diplomatisch und nicht polarisierend.

\section{Gruppenidentifikationsfaktor Nummer 6: Individualität wahren!}

Je größer die Cruppe, umso schwieriger ist die Einflussnahme auf bzw. die Kommunikation mit der Gruppe. Abgesehen von den notwendigen Kollektivansprachen sollte der innerliche Fokus des Managements aber immer (wieder) auf die individuelle Ansprache gerichtet sein.

- Jedes Verbundmitglied bedarf der individuellen Betreuung und Ansprache.

- Lob und Anerkennung sollten ein Selbstverständnis sein.

- Keine Anfrage darf unbeantwortet bleiben.

- Kritik ist vielleicht nicht schön, aber notwendig und ernstzunehmen!

- Netzmitglieder sollten im Rahmen ihrer Kompetenzen um Rat und zu konkreten Vorschlägen befragt werden.

\section{Gruppenidentifikationsfaktor Nummer 7: Abkehr vermeiden!}

Auch gewonnene Netzmitglieder wollen gepflegt werden. Hier die drei Todsünden der Netzarbeit.

- Vermeidung unnötiger Informationsflut. Der Netzarzt schaltet ab.

- Verhinderung von Individualitätsverlust. Der Netzarzt fühlt sich nicht mehr angesprochen.

- Vorbeugung von Unruhe durch intransparente Veränderungen. Der Netzarzt verliert das Vertrauen in die Organisation. 


\section{Gruppenidentifikationsfaktor Nummer 8: Image pflegen!}

Kennt jemand anders den Verbund außer den Mitgliedern selbst? Und was steckt eigentlich inhaltlich hinter Abkürzungen und Rechtsformen von Ärztenetzen? Hier nur einige von denkbaren Spiegelstrichen, wobei die Reihenfolge nicht willkürlich ist:

- Die ersten beiden Punkte sind konstituierende Faktoren, denn es muss analysiert werden, was der Markt erfordert und was das Netz diesen Nachfragern seinerseits anbieten möchte. Hier muss Konsens erzielt werden!

- Identifikation der Bedürfnisse der Kunden des Netzes, als da sind: Patienten, Krankenhäuser, Krankenkassen und potenzielle neue Mitglieder

- eindeutige Formulierung gemeinsamer Verbundziele (Corporate Identity)

- Fassbarkeit des virtuellen Verbundes durch konkrete Projektierungen wie z. B. Gründung einer gemeinsamen Notfallambulanz, eines Netzbüros, einer Physiotherapie. Der Kreativität sind dabei kaum Grenzen gesetzt.

- Regelmäßige Arbeiten am Image des Netzes durch regionale Markenbildung. Das Thema Gesundheit sollte automatisch in Zusammenhang mit dem Namen des Verbundes gebracht werden.

- Gründung von Kooperationen oder eigenen Unternehmen im Sinne einer ganzheitlichen Betreuung der Patienten mit anderen Professionen wie Therapeuten, Ernährungsberatern, etc.

- Corporate Design, z. B. jede beteiligte Praxis trägt das Verbundlogo an der Tür

- Stete Berichte über Erfolge der Netzarbeit durch Angebote zur Gesunderhaltung der Bevölkerung auf der eigenen Homepage, in der lokalen Presse und der überregionalen Fachpresse

Teilnahme an Wettbewerben und Ausschreibungen

\section{Gruppenidentifikationsfaktor Nummer 9: Sanktionen formulieren!}

In allen Gruppen gibt es immer vier Verhaltensweisen des einzelnen Gruppenmitglieds gegenüber den Anforderungen und Sachzwängen der Gruppe: erforderliches, erwünschtes, erlaubtes und verbotenes Verhalten. Diese Verhaltensweisen sollte die Gruppe gemäß ihren Normen mit Belohnung oder Bestrafung sanktionieren, denn die Verinnerlichung von Normen machen die Anwendung äußerer Sanktionen nicht überflüssig. (Definition von Normen mit Sanktionen in Netzkodexen, Satzungen oder Leitbildern.)

Wie ausgeführt, ist dieser Maßnahmenkatalog dem Tagesgeschäft des Ärztenetzes Medizin und Mehr in den letzten 11 Jahren erwachsen und erhebt keinen Anspruch auf Vollständigkeit. Vielmehr ist es Aufgabe und Herausforderung im Sinne einer lernenden Organisation, diesen ständig zu bearbeiten und zu erweitern. 